\title{
On the String Equation of the BKP Hierarchy
}

\author{
Hsin-Fu Shen ${ }^{1}$ and Ming-Hsien $\mathrm{Tu}^{2} *$ \\ ${ }^{1}$ Department of Mechanical Engineering, WuFeng Institute of Technology, \\ Chiayi 621, Taiwan, \\ 2 Department of Physics, National Chung Cheng University, \\ Chiayi 621, Taiwan
}

November 3, 2018

\begin{abstract}
The Adler-Shiota-van Moerbeke formula is employed to derive the $W$-constraints for the $p$-reduced BKP hierarchy constrained by the string equation. We also provide the Grassmannian description of the string equation in terms of the spectral parameter.

PACS: 02.30.Ik

Keywords: BKP hierarchy, additional symmetries, vertex operators, string equation, $W$ constraints, Sato Grassmannian.
\end{abstract}

*E-mail: phymhtu@ccu.edu.tw 


\section{Introduction}

String equation is an important constrained condition connecting integrable hierarchies with solvable string theories and intersection theory (see e.g. [8, 28, and references therein). The most famous example is the KdV hierarchy constrained by a string equation whose solutions corresponds to partition function of $2 \mathrm{~d}$ quantum gravity or generating function of intersection number [29, 30, 15] (see also [13]). Since the solutions of KdV hierarchy can be characterized by a single function called $\tau$-function, it turns out that the constrained KdV hierarchy can be written as Virasoro constraints on the $\tau$-function, in which the lowest one corresponds to the string equation [9, 11, 16, 17]. For higher order KdV hierarchy (i.e. p-reduced KP hierarchy with $p>2$ ) the associated $\tau$-function satisfies the $W$-constraints so that the $\tau$-function is a null-vector of a set of $W_{p}^{+}$-algebra which contains the Virasoro algebra as a subalgebra with central charge $c=p[10,1,28$. It was pointed out [16, 17, 5] that the string equation associated with the $\mathrm{KP} / \mathrm{KdV}$ hierarchy can be traced back to additional symmetries (or non-isospectral flows )of the hierarchies proposed by Orlov and Schulman[18](see also [7]). Furthermore, Adler-Shiota-van Moerbeke [2, 3] and Dickey [6] showed that additional symmetries acting on wave function are connected with Sato's Bäcklund symmetries acting on tau function. These properties provide a useful tool to study the solutions to the string equation of the $p$-reduced KP hierarchy with $p>2[1$ that generalize Kontsevich's result for the KdV case.

In the present work, we like to study the string equation associated with the BKP hierarchy 4 , 24. The BKP hierarchy possesses many integrable structures as the KP hierarchy such as Lax formulation, tau function, Hirota bilinear equation, fermion representation, soliton and quasiperiodic solutions, etc. In [26] van de Leur provided the Adler-Shiota-van Moerbeke formula for the BKP hierarchy. He also studied the $W$-constraint for the $p$-reduced BKP hierarchy in terms of the twisted affine Lie algebra $\hat{s l}_{n}^{2}[27$. In this work, we follow closely the work by Adler-van Moerbeke[1] and Dickey [5] to reconsider the string equation of the BKP hierarchy from the point of view of additional symmetries in Lax-Orlov-Schulman formulation. In particular, we shall show that the invariance of the $p$-reduced BKP hierarchy with respect to a particular additional symmetry is crucial for obtaining the string equation which together with the Adler-Shiota-van Moerbeke formula implies the $W$-constraints of the $\tau$ function. In the end of the work we also give a Grassmannian description of the string equation in terms of the spectral parameter.

The paper is organized as follows. In section 2, we recall some basic notions for the BKP hierarchy such as Lax formulation, (adjoint-) wave function, $\tau$ function and (differential) Fay identity. In section 3, we introduce the Orlov-Schulman operator for additional symmetries 
and vertex operators for Bäcklund symmetries of the BKP hierarchy. We give a simple expression of the generators of vertex operator using the Faà di Bruno polynomials and provide the Adler-Shiota-van Moerbeke formula that connects additional symmetries acting on wave function with those Sato's Bäcklund symmetries acting on tau function. In section 4, we show that the solution of the $p$-reduced BKP hierarchy constrained by the string equation can be characterized by a vacuum condition so that the associated tau function is annihilated by a set of differential operators. In section 5 , We show that these differential operators constitute a $W_{p}^{B+}$ algebra which contains a Virasoro algebra as a subalgebra with central charge $c=p$. In section 6 , we provide the Grassmannian description of the string equation in terms of the spectral parameter. Section 7 is devoted to the concluding remarks.

\section{BKP hierarchy}

In this section we recall some basic properties for the BKP hierarchy [4]. We shall follow the notations used in the previous work[25]. The BKP hierarchycan be formulated in Lax form as

$$
\partial_{2 n+1} L=\left[B_{2 n+1}, L\right], \quad B_{2 n+1}=\left(L^{2 n+1}\right)_{+}, \quad n=0,1,2, \cdots
$$

where the Lax operator is defined by

$$
L=\partial+u_{1} \partial^{-1}+u_{2} \partial^{-2}+\cdots,
$$

with coefficient functions $u_{i}$ depending on the time variables $t=\left(t_{1}=x, t_{3}, t_{5}, \cdots\right)$ and satisfies the constraint

$$
L^{*}=-\partial L \partial^{-1}
$$

Here and the rest of the paper we will use the notations: $\left(\sum_{i} a_{i} \partial^{i}\right)_{+}=\sum_{i \geq 0} a_{i} \partial^{i},\left(\sum_{i} a_{i} \partial^{i}\right)_{-}=$ $\sum_{i<0} a_{i} \partial^{i},\left(\sum_{i} a_{i} \partial^{i}\right)_{[k]}=a_{k}, \operatorname{res}\left(\sum_{i} a_{i} \partial^{i}\right)=a_{-1}$ and $\left(\sum_{i} a_{i} \partial^{i}\right)^{*}=\sum_{i}(-\partial)^{i} a_{i}$. It can be shown [4] that the constraint (3) is equivalent to the condition $\left(B_{2 n+1}\right)_{[0]}=0$.

The Lax equation (11) is equivalent to the compatibility condition of the linear system

$$
L w(t, z)=z w(t, z), \quad \partial_{2 n+1} w(t, z)=B_{2 n+1} w(t, z),
$$

where $w(t, z)$ is called wave function (or Baker function) of the system and $z$ is the spectral parameter. The whole hierarchy can be expressed in terms of a dressing operator, the so-called Sato's operator $W$, so that

$$
L=W \partial W^{-1}, \quad W=1+\sum_{j=1} w_{j} \partial^{-j},
$$


and the Lax equation is equivalent to the Sato's equation

$$
\partial_{2 n+1} W=-\left(L^{2 n+1}\right)_{-} W
$$

with constraint 23,24$]$

$$
W^{*} \partial W=\partial .
$$

Let the solutions of the linear system (4) be the form

$$
w(t, z)=W e^{\xi(t, z)}=\hat{w}(t, z) e^{\xi(t, z)},
$$

where $\xi(t, z)=\sum_{i=0} t_{2 i+1} z^{2 i+1}$ and $\hat{w}(t, z)=1+w_{1} / z+w_{2} / z^{2}+\cdots$. Then $w(t, z)$ is a wave function of the BKP hierarchy if and only if it satisfies the bilinear identity 4

$$
\operatorname{res}_{z}\left(z^{-1} w(t, z) w\left(t^{\prime},-z\right)\right)=1, \quad \forall t, t^{\prime}
$$

where we denote the $\operatorname{symbol} \operatorname{res}_{z}\left(\sum_{i} a_{i} z^{i}\right)=a_{-1}$. In fact, from the bilinear identity (8), solutions of the BKP hierarchy can be characterized by a single function $\tau(t)$ called $\tau$-function such that 4

$$
\hat{w}(t, z)=\frac{\tau\left(t_{1}-\frac{2}{z}, t_{3}-\frac{2}{3 z^{3}}, t_{5}-\frac{2}{5 z^{5}}, \cdots\right)}{\tau(t)} .
$$

From (7) and (9) the wave function $w(t, \lambda)$ can be expressed in terms of $\tau$-function as

$$
w(t, z)=\frac{X_{B}(t, z) \tau(t)}{\tau(t)},
$$

where $X_{B}(t, z)$ is the so-called vertex operator, defined by [4]

$$
X_{B}(t, z)=e^{\xi(t, z)} e^{-2 D(t, z)} \equiv e^{\xi(t, z)} G(z),
$$

with $D(t, z)=\sum_{n=0} z^{-2 n-1} \partial_{2 n+1} /(2 n+1)$.

In the following we provide two useful identities associated with the tau function of the BKP hierarchy.

Proposition 1. [25] (Fay identity) The tau function of the BKP hierarchy satisfies the Fay quadrisecant identity:

$$
\begin{aligned}
& \sum_{\left(s_{1}, s_{2}, s_{3}\right)} \frac{\left(s_{1}-s_{0}\right)\left(s_{1}+s_{2}\right)\left(s_{1}+s_{3}\right)}{\left(s_{1}+s_{0}\right)\left(s_{1}-s_{2}\right)\left(s_{1}-s_{3}\right)} \tau\left(t+2\left[s_{2}\right]+2\left[s_{3}\right]\right) \tau\left(t+2\left[s_{0}\right]+2\left[s_{1}\right]\right) \\
& +\frac{\left(s_{0}-s_{1}\right)\left(s_{0}-s_{2}\right)\left(s_{0}-s_{3}\right)}{\left(s_{0}+s_{1}\right)\left(s_{0}+s_{2}\right)\left(s_{0}+s_{3}\right)} \tau\left(t+2\left[s_{0}\right]+2\left[s_{1}\right]+2\left[s_{2}\right]+2\left[s_{3}\right]\right) \tau(t)=0
\end{aligned}
$$

where $\left(s_{1}, s_{2}, s_{3}\right)$ stands for cyclic permutations of $s_{1}, s_{2}$ and $s_{3}$. 
Proposition 2. [25] (Differential Fay identity) The following equation holds.

$$
\begin{aligned}
& \left(\frac{1}{s_{2}^{2}}-\frac{1}{s_{1}^{2}}\right)\left\{\tau\left(t+2\left[s_{1}\right]+2\left[s_{2}\right]\right) \tau\left(t+2\left[s_{2}\right]\right)-\tau\left(t+2\left[s_{1}\right]+2\left[s_{2}\right]\right) \tau(t)\right\} \\
& =\left(\frac{1}{s_{2}}+\frac{1}{s_{1}}\right)\left\{\partial \tau\left(t+2\left[s_{2}\right]\right) \tau\left(t+2\left[s_{1}\right]\right)-\partial \tau\left(t+2\left[s_{1}\right]\right) \tau\left(t+2\left[s_{2}\right]\right)\right\} \\
& \quad+\left(\frac{1}{s_{2}}-\frac{1}{s_{1}}\right)\left\{\tau\left(t+2\left[s_{1}\right]+2\left[s_{2}\right]\right) \partial \tau(t)-\partial \tau\left(t+2\left[s_{1}\right]+2\left[s_{2}\right]\right) \tau(t)\right\}
\end{aligned}
$$

\section{Additional symmetries and vertex operators}

Based on the work of Orlov and Schulman [18, the Lax equation can be extended by introducing the Orlov-Schulman operator $M$ defined by

$$
M=W \Gamma W^{-1}, \quad \Gamma=\sum_{n=0}(2 n+1) t_{2 n+1} \partial^{2 n}
$$

which satisfies

$$
\partial_{2 n+1} M=\left[B_{2 n+1}, M\right], \quad[L, M]=1 .
$$

Thus the linear system (4) should be extended to

$$
L w=z w, \quad M w=\partial_{z} w, \quad \partial_{2 n+1} w=B_{2 n+1} w .
$$

Note that on the space of wave function $w(t, z),(L, M)$ is anti-isomorphic to $\left(z, \partial_{z}\right)$ since $\left[z, \partial_{z}\right]=-1$. More general, one has

$$
M^{m} L^{l} w=z^{l} \partial_{z}^{m} w, \quad L^{l} M^{m} w=\partial_{z}^{m} z^{l} w
$$

In fact, one can define the adjoint wave function $w^{*}(t, z)=\left(W^{*}\right)^{-1} e^{-\xi(t, z)}=-z^{-1} w_{x}(t,-z)$ and $M^{*}=\left(L^{*}\right)^{-1} \partial M \partial^{-1} L^{*}$ where we have use the fact that $\Gamma^{*}=\Gamma$ and (6). Then $\left[L^{*}, M^{*}\right]=$ $[M, L]^{*}=-1$, and

$$
L^{*} w^{*}=z w^{*}, \quad M^{*} w^{*}=-\partial_{z} w^{*}, \quad \partial_{2 n+1} w^{*}=-B_{2 n+1}^{*} w^{*} .
$$

Motivated by the KP hierarchy, one can introduce a new set of parameters $\hat{t}_{m l}$ so that additional symmetries of the BKP hierarchy can be expressed as

$$
\hat{\partial}_{m l} W=-\left(A_{m l}(L, M)\right)_{-} W
$$

where the generator $A_{m l}(L, M)$, due to (6), has the form [26, 25]

$$
A_{m l}(L, M)=M^{m} L^{l}-(-1)^{l} L^{l-1} M^{m} L .
$$


Let us introduce another generator $Y_{B}(\lambda, \mu)$ of additional symmetries as [26]

$$
\begin{aligned}
Y_{B}(\lambda, \mu) & =\sum_{m=0}^{\infty} \frac{(\mu-\lambda)^{m}}{m !} \sum_{l=-\infty}^{\infty} \lambda^{-l-m-1}\left(A_{m, m+l}(L, M)\right)_{-}, \\
& =\sum_{m=0}^{\infty} \frac{(\mu-\lambda)^{m}}{m !} \sum_{l=-\infty}^{\infty} \lambda^{-l-m-1}\left(M^{m} L^{m+l}-(-1)^{m+l} L^{m+l-1} M^{m} L\right)_{-} .
\end{aligned}
$$

We mention that for $\lambda=\mu$, the generator $Y_{B}(\lambda, \lambda)=2 \sum_{l=o d d} \lambda^{-l-1} L_{-}^{l}$ corresponds to the resolvent operator of the BKP hierarchy.

On the other hand, recalling the vertex operator $X_{B}(\lambda, \mu)$ defined by

$$
X_{B}(\lambda, \mu)=e^{-\xi(t, \lambda)} e^{\xi(t, \mu)} G(-\lambda) G(\mu)
$$

which provides the infinitesimal Sato's Bäcklund transformations 4 , on the space of tau function, namely, if $\tau(t)$ is a solution then $\tau(t)+\epsilon X_{B}(\lambda, \mu) \tau(t)$ is a solution as well. In fact, one can Taylor expand the vertex operator $X_{B}(\lambda, \mu)$ around $\mu=\lambda$ for large $\lambda$ as

$$
X_{B}(\lambda, \mu)=\sum_{m=0} \frac{(\mu-\lambda)^{m}}{m !} W^{(m)}(\lambda)=\sum_{m=0} \frac{(\mu-\lambda)^{m}}{m !} \sum_{l=-\infty}^{\infty} \lambda^{-m-l} W_{l}^{(m)},
$$

where $W^{(m)}(\lambda)=\left.\partial_{\mu}^{m} X_{B}(\lambda, \mu)\right|_{\mu=\lambda}$. Introducing the symbol $\alpha(z)=\sum_{n=o d d} \alpha_{n} z^{-n} / n$ with

$$
\alpha_{n}= \begin{cases}2 \partial / \partial t_{n} & n \in \mathbb{Z}_{\text {odd }}^{+} \\ |n| t_{|n|} & n \in \mathbb{Z}_{\text {odd }}^{-}\end{cases}
$$

where $\mathbb{Z}_{\text {odd }}=\mathbb{Z}_{\text {odd }}^{+} \oplus \mathbb{Z}_{\text {odd }}^{-}$. Then $\alpha_{n}$ 's satisfy the commutation relations

$$
\left[\alpha_{n}, \alpha_{m}\right]=2 n \delta_{n,-m}, \quad n \in \mathbb{Z}_{\text {odd }} .
$$

The vertex operator $X_{B}(\lambda, \mu)$ can be expressed as

$$
X_{B}(\lambda, \mu)=: e^{\alpha(\lambda)-\alpha(\mu)}:
$$

where the normal ordering :: demands that $\alpha_{n>0}$ must be placed to the right of $\alpha_{n<0}$. Therefore,

$$
W^{(m)}(\lambda)=\left.\partial_{\mu}^{m} X_{B}(\lambda, \mu)\right|_{\mu=\lambda}=: \partial_{\lambda}^{m} e^{-\alpha(\lambda)} \cdot e^{\alpha(\lambda)}:=: F_{m}\left(-\partial_{\lambda} \alpha(\lambda)\right):
$$

where $F_{m}(u(z))$ is the so-called Faà di Bruno polynomials (see e.g. [7]) defined by the recurrence relations $F_{m+1}(u)=\left(\partial_{z}+u\right) F_{m}(u)$. For instance, $F_{0}=1, F_{1}=u, F_{2}=u^{\prime}+u^{2}, F_{3}=u^{\prime \prime}+$ $3 u u^{\prime}+u^{3}$.

Lemma 3. [6] The following formula

$$
W^{(m)}(\lambda)=\sum_{m_{1}+2 m_{2}+\cdots=m} \frac{m !}{m_{1} ! m_{2} ! \cdots}:\left(-\frac{\partial_{\lambda} \alpha}{1 !}\right)^{m_{1}}\left(-\frac{\partial_{\lambda}^{2} \alpha}{2 !}\right)^{m_{2}} \cdots:
$$

holds, where $m_{i} \geq 0$. 
Proof. Introducing a generating function of $F_{m}$ as $g(\lambda, z)=\sum_{m=0} F_{m} z^{m} / m$ !, then

$$
\begin{aligned}
g(\lambda, z) & =\sum_{m=0} \frac{\left(d_{\lambda}^{m} 1\right)}{m !} z^{m}, \quad d_{\lambda}=e^{\alpha(\lambda)} \cdot \partial_{\lambda} \cdot e^{-\alpha(\lambda)} \\
& =e^{\alpha(\lambda)} \cdot e^{z \partial_{\lambda}} e^{-\alpha(\lambda)} \\
& =e^{\alpha(\lambda)} e^{-\alpha(\lambda+z)} \\
& =\prod_{n=1} e^{-\partial_{\lambda}^{n} \alpha z^{n} / n !} \\
& =\sum_{m_{1}=0} \frac{\left(-\frac{\partial_{\lambda} \alpha}{1 !}\right)^{m_{1}} z^{m_{1}}}{m_{1} !} \sum_{m_{2}=0} \frac{\left(-\frac{\partial_{\lambda}^{2} \alpha}{2 !}\right)^{m_{2}} z^{2 m_{2}}}{m_{2} !} \cdots \\
& =\sum_{m=0}\left(\sum_{m_{1}+2 m_{2}+\cdots=m} m ! \frac{\left(-\frac{\partial_{\lambda} \alpha}{1 !}\right)^{m_{1}}}{m_{1} !} \frac{\left(-\frac{\partial_{\lambda}^{2} \alpha}{2 !}\right)^{m_{2}}}{m_{2} !} \cdots\right) \frac{z^{m}}{m !}
\end{aligned}
$$

The vertex operator generators $W_{l}^{(m)}$ can be easily computed as

$$
\begin{aligned}
W_{n}^{(0)} & =\delta_{n, 0}, \\
W_{n}^{(1)} & =\left\{\begin{array}{ll}
\alpha_{n} & n \in \mathbb{Z}_{\text {odd }} \\
0 & n \in \mathbb{Z}_{\text {even }}
\end{array},\right. \\
W_{n}^{(2)} & =\left\{\begin{array}{ll}
-(n+1) \alpha_{n} & n \in \mathbb{Z}_{\text {odd }} \\
\sum_{i+j=n}: \alpha_{i} \alpha_{j}: & n \in \mathbb{Z}_{\text {even }}
\end{array},\right. \\
W_{n}^{(3)}= & \left\{\begin{array}{ll}
\sum_{i+j+k=n}: \alpha_{i} \alpha_{j} \alpha_{k}:+(n+1)(n+2) \alpha_{n} & n \in \mathbb{Z}_{\text {odd }} \\
-\frac{3}{2}(n+2) \sum_{i+j=n}: \alpha_{i} \alpha_{j}: & n \in \mathbb{Z}_{\text {even }}
\end{array},\right. \\
W_{n}^{(4)}= & \begin{cases}-2(n+3) \sum_{i+j+k=n}: \alpha_{i} \alpha_{j} \alpha_{k}:-(n+1)(n+2)(n+3) \alpha_{n} & n \in \mathbb{Z}_{\text {odd }} \\
\sum_{i+j+k+l=n}: \alpha_{i} \alpha_{j} \alpha_{k} \alpha_{l}:-\sum_{i+j=n} i j: \alpha_{i} \alpha_{j}: & n \in \mathbb{Z}_{\text {even }} \\
-\left(2 n^{2}+9 n+11\right) \sum_{i+j=n}: \alpha_{i} \alpha_{j}: & \end{cases}
\end{aligned}
$$

etc.

A remarkable formula described below provides a bridge between additional symmetries acting on wave function and Sato's Bäcklund symmetries acting on $\tau$ function. This kind of formula was first derived for the KP hierarchy by Adler-Shiota-van Moerbeke 2, 3] and Dickey[6], and later for BKP by van de Leur[26] (see also [25]).

Theorem 4. [26, 25] The following formula

$$
X_{B}(\lambda, \mu) w(t, z)=2 \lambda\left(\frac{\lambda-\mu}{\lambda+\mu}\right) Y_{B}(\lambda, \mu) w(t, z)
$$


holds for the BKP hierarchy, where it should be understood that the vertex operator $X_{B}(\lambda, \mu)$ acting on $w(t, z)$ is generated by its action on the $\tau$ function.

We remark that the proof of (14) in [25] is based on a simple expression for the generator $Y_{B}(\lambda, \mu)$ and the differential Fay identity of the BKP hierarchy. Through the fermion-boson correspondence in the BKP hierarchy, a realization of Lie algebra $g o(\infty)$ on $\mathbb{C}\left[t_{1}, t_{3}, t_{5}, \cdots\right]$ is given by 4

$$
Z_{B}(\lambda, \mu)=\frac{1}{2} \frac{\mu+\lambda}{\mu-\lambda}\left(X_{B}(\lambda, \mu)-1\right)
$$

which after Taylor expanding around $\mu=\lambda$ for large $\lambda$ has the form

$$
Z_{B}(\lambda, \mu)=\sum_{m=0} \frac{(\mu-\lambda)^{m}}{m !} \sum_{l=-\infty}^{\infty} \lambda^{-m-l} Z_{l}^{(m+1)} .
$$

It is easy to show that differential operators $Z_{l}^{(m)}$ are related to $W_{l}^{(m)}$ as

$$
Z_{l}^{(1)}=W_{l}^{(1)}, \quad Z_{l}^{(m+1)}=\frac{W_{l}^{(m+1)}}{m+1}+\frac{1}{2} W_{l}^{(m)}, \quad m \geq 1,
$$

and constitute an infinite-dimensional Lie algebra called $W_{1+\infty}^{B}$-algebra which is a subalgebra of $W_{1+\infty}$ associated with the KP hierarchy.

\section{$4 p$-reduced BKP and string equation}

The so-called $p$-reduced BKP hierarchy[4] is defined by the Lax operator (2) such that

$$
L^{p}=\left(L^{p}\right)_{+}, \quad p=\text { odd integer }
$$

This reduction must be compatible with the condition (3). For example, $\left(L^{p}\right)_{[0]}=0$ for odd $p$ and thus

$$
\left(L^{p}\right)_{+}^{*}=\left(L^{*}\right)^{p}=(-1)^{p} \partial L^{p} \partial^{-1}=(-1)^{p} \partial\left(L^{p}\right)_{+} \partial^{-1}
$$

is a differential operator as well. Therefore, from the Lax equation (1), we have

$$
\partial_{2 n+1} L^{p}=\left[\left(L^{p}\right)_{+}^{\frac{2 n+1}{p}}, L^{p}\right]
$$

which implies that $L^{p}$ is independent of the parameters $t_{j p}$ for $j=1,3,5, \cdots$. For $p=1$ case, we have $L=L_{+}=\partial$ which is a trivial reduction $\left(u_{i}=0\right.$ for $\left.\forall i\right)$. The next case is $p=3$, which contains the simplest nontrivial equation called Sawada-Kotera equation[20]:

$$
u_{t}+15\left(u^{3}+u u_{x x}\right)_{x}+u_{x x x x x}=0
$$

where $x=t_{1}, t=t_{5}$ and $u=2(\log \tau)_{x x}$ with $\partial \tau / \partial t_{3 j}=0(j=1,3,5, \cdots)$. 
In the following, we would like to characterize the solutions of the $p$-reduced BKP hierarchy constrained by the string equation

$$
\left[L^{p}, P\right]=1
$$

where $P$ is a differential operator. From (11), we have

$$
A_{1,1-k}= \begin{cases}k L^{-k} & k \in \mathbb{Z}_{\text {odd }} \\ 2 M L^{1-k}-k L^{-k} & k \in \mathbb{Z}_{\text {even }}\end{cases}
$$

Guided by the KP hierarchy [5], if we think the string equation (17) as the consequence of the addition flow equation $\hat{\partial}_{1,1-p} W=0$ for odd $p$, then (10) and (18) show that $\left(A_{1,1-p}\right)_{-}=$ $p L^{-p}=0$ which produces a contradictory result. $\operatorname{In}[27$ van de Luer pointed out that one may consider the additional flow equation $\hat{\partial}_{1,1-2 p} W=-\left(A_{1,1-2 p}\right)_{-} W=0$ from which one gets

$$
\left(M L^{1-2 p}\right)_{-}=p L^{-2 p}
$$

and hence the operator $Q \equiv\left(M L^{1-2 p}-p L^{-2 p}\right) / 2 p$ is purely differential. Then

$$
\left[L^{2 p}, Q\right]=1
$$

However, in view of the fact that $L^{p}=\left(L^{p}\right)_{+}$, we have

$$
\left(M L^{1-p}\right)_{-}=\left(M L^{1-2 p} L^{p}\right)_{-}=p L^{-p}
$$

which provides the differential operator $P \equiv\left(M L^{1-p}-p L^{-p}\right) / p$ for the string equation (17). Therefore, (19) can be regarded as the symmetry origin of the string equation (17), and thus we refer (19) to the pre-string equation. Taking the residue of (19) we obtain

$$
\sum_{n=p}(2 n+1) t_{2 n+1} \operatorname{res}\left(L^{2 n-2 p+1}\right)+(2 p-1) t_{2 p-1}=0 .
$$

From the Sato's equation (5) and the formula (91), we have

$$
\operatorname{res}\left(L^{2 n-2 p+1}\right)=2 \partial_{1} \partial_{2 n-2 p+1} \log \tau .
$$

Substituting above back to (22) and integrating it over $t_{1}$, yields

$$
\left(\sum_{n=p}(2 n+1) t_{2 n+1} \frac{\partial}{\partial t_{2 n+1-2 p}}+\frac{(2 p-1)}{2} t_{1} t_{2 p-1}+c\right) \tau(t)=0 .
$$

In fact, from (19) and the $p$-reduced BKP hierarchy flows, we can prove a more general result of constraints on $\tau$-function. 
Proposition 5. For the p-reduced Lax operator $L^{p}$ constrained by the string equation, the following formulas hold for $m \geq 0$.

$$
\begin{aligned}
\left(M^{m} L^{j p+m}\right)_{-} & = \begin{cases}\prod_{r=0}^{m-1}(p-r) L^{-2 p} & j=-2 \\
\prod_{r=0}^{m-1}(p-r) L^{-p} & j=-1 \\
0 & j=0,1,2, \ldots\end{cases} \\
\left(L^{j p+m-1} M^{m} L\right)_{-} & =(-1)^{m}\left(M^{m} L^{j p+m}\right)_{-}
\end{aligned}
$$

with the proviso that the factor $\prod_{r=0}^{m-1}(p-r)$ should be set to 1 for $m=0$.

Proof. We shall follow [1] to prove it by induction on $m$ and $j$. For $m=0$, the proof is obvious. For $m=1, j=-2$, this is just the equation (19). Assume that the formula (24) holds up to some integer $m>0$ for $j=-2$, then for $j \geq-1$, one sees that

$$
\begin{aligned}
\left(M^{m} L^{j p+m}\right)_{-} & =\left(\left(M^{m} L^{m-2 p}\right)_{-} L^{(j+2) p}\right)_{-} \\
& =\left(\prod_{r=0}^{m-1}(p-r) L^{-2 p} L^{(j+2) p}\right)_{-} \\
& = \begin{cases}\prod_{r=0}^{m-1}(p-r) L^{-p} & j=-1 \\
0 & j=0,1,2, \ldots\end{cases}
\end{aligned}
$$

Next, for the $j=-2$, we have

$$
\begin{aligned}
\left(M^{m+1} L^{m+1-2 p}\right)_{-} & =\left(M^{m} M L^{m} L^{1-2 p}\right)_{-} \\
& =\left(M^{m} L^{m} M L^{1-2 p}\right)_{-}-m\left(M^{m} L^{m-2 p}\right)_{-} \\
& =\left(M^{m} L^{m}\left(M L^{1-2 p}\right)_{-}\right)_{-}-m\left(M^{m} L^{m-2 p}\right)_{-} \\
& =p\left(M^{m} L^{m-2 p}\right)_{-}-m\left(M^{m} L^{m-2 p}\right)_{-} \\
& =(p-m) \prod_{r=0}^{m-1}(p-r) L^{-2 p} \\
& =\prod_{r=0}^{m}(p-r) L^{-2 p}
\end{aligned}
$$

where the third equality is due to the fact that $M^{m} L^{m}$ is a differential operator. The formula (25) can be proved in a similar way.

We like to mention that a similar result as (24) for the CKP hierarchy has been derived in [12] to discuss the associated additional symmetries and string equation.

Proposition 6. Let $L^{p}$ be the Lax operator of the p-reduced BKP hierarchy constrained by the string equation (17). Then for $m \geq 0$ and $j \geq-2$,

$$
Z_{j p}^{(m+1)} \tau(t)=c \cdot \tau(t)
$$


where $c$ is a constant.

Proof. Using (12) and the Adler-Shiota-van Moerbeke formula (14), we have

$$
\begin{aligned}
\left(A_{m, m+j p}\right)_{-} \omega(t, z) & =\operatorname{res}_{\lambda}\left(\left.\lambda^{j p+m} \partial_{\mu}^{m}\right|_{\mu=\lambda} Y_{B}(\lambda, \mu) \omega(t, z)\right) \\
& =\operatorname{res}_{\lambda}\left(\left.\lambda^{j p+m-1} \partial_{\mu}^{m}\right|_{\mu=\lambda} \frac{1}{2} \frac{\lambda+\mu}{\lambda-\mu} X_{B}(\lambda, \mu) \omega(t, z)\right) \\
& =-\omega(t, z)(G(z)-1) \operatorname{res}_{\lambda}\left(\left.\lambda^{j p+m-1} \partial_{\mu}^{m}\right|_{\mu=\lambda} \frac{Z_{B}(\lambda, \mu) \tau(t)}{\tau(t)}\right) \\
& =-\omega(t, z)(G(z)-1)\left(\frac{Z_{j p}^{(m+1)} \tau(t)}{\tau(t)}\right) .
\end{aligned}
$$

On the other hand, from (24) and (25) we have

$$
\begin{aligned}
\left(A_{m, m+j p}\right)_{-} \omega(t, z) & =\left(\left(M^{m} L^{j p+m}\right)_{-}-(-1)^{j p+m}\left(L^{j p+m-1} M^{m} L\right)_{-}\right) \omega(t, z) \\
& =\left(1-(-1)^{j p}\right)\left(M^{m} L^{j p+m}\right)_{-} \omega(t, z) \\
& =2 \prod_{r=0}^{m-1}(p-r) L^{-p} \omega(t, z) \delta_{j,-1} \\
& =2 \prod_{r=0}^{m-1}(p-r) z^{-p} \omega(t, z) \delta_{j,-1}
\end{aligned}
$$

Noticing that

$$
2 z^{-p}=-\left(p\left(t_{p}-2 z^{-p} / p\right)-p t_{p}\right)=-(G(z)-1) \frac{Z_{-p}^{(1)} \tau(t)}{\tau(t)}
$$

and hence

$$
(G(z)-1)\left(\frac{Z_{j p}^{(m+1)} \tau(t)}{\tau(t)}-\prod_{r=0}^{m-1}(p-r) \delta_{j,-1} \frac{Z_{-p}^{(1)} \tau(t)}{\tau(t)}\right)=0 .
$$

Since $(G(z)-1) f(t)=0$ implies $f(t)$ is a constant, we have

$$
\left(Z_{j p}^{(m+1)}-\prod_{r=0}^{m-1}(p-r) \delta_{j,-1} Z_{-p}^{(1)}\right) \tau(t)=c \cdot \tau(t) .
$$

Now we can drop the term involving $Z_{-p}^{(1)}=p t_{p}$ without harm because the $p$-reduced BKP hierarchy does not depend on the variables $t_{p}$.

\section{String equation as the lowest Virasoro constraint}

In this section, we like to discuss the algebraic structure of the equation (26). Given a infinitedimensional algebra $W_{1+\infty}^{B}$ defined by the vertex operator, one can introduce two subalgebras as follows:

$$
W_{p}^{B}=\left\{\text { generated by } W_{j p}^{(m)}, 1 \leq m \leq p, j \in \mathbb{Z}, t_{p}=t_{3 p}=\cdots=0\right\}
$$


and its truncated sub-algebra:

$$
W_{p}^{B+}=\left\{\text { generated by } W_{j p}^{(m)}, 1 \leq m \leq p, j \geq-2, t_{p}=t_{3 p}=\cdots=0\right\}
$$

We shall show that the $\tau$ function satisfying the $p$-reduced BKP hierarchy and the string equation is a null-vector of the $W_{p}^{B+}$-algebra. Since the algebra $W_{p}^{+}$for the KP hierarchy has no central term[10], thus we expect that the subalgebra $W_{p}^{B+} \subset W_{p}^{+}$has also no central term. To see this, we have to properly combine the generators $Z_{j p}^{(m)}$ so that every redefined element $\mathbf{W}_{n}^{(m)}$ of $W_{p}^{B+}$ can be expressed as a commutator of two elements of $W_{p}^{B+}$. As a consequence, the constant $c$ in (26) can be removed and

$$
\mathbf{W}_{n}^{(m)} \tau(t)=0, \quad 1 \leq m \leq p, n \geq-m+1 .
$$

We remark that the condition for the subscript $n$ in (27) is due to the fact that for higher-spin generators $\mathbf{W}_{n}^{(m)}$, one has

$$
\left[\mathbf{W}_{-1}^{(2)}, \mathbf{W}_{n}^{(m)}\right]=(-(m-1)-n) \mathbf{W}_{n-1}^{(m)},
$$

and thus, under bracketing with generators $\mathbf{W}_{-1}^{(2)}$, one can reach the lowest one $\mathbf{W}_{-m+1}^{(m)}$ which commutes with $\mathbf{W}_{-1}^{(2)}$. Let us demonstrate the first few $W$-constraints.

For $m=0,(26)$ shows that

$$
Z_{j p}^{(1)} \tau(t)=W_{j p}^{(1)} \tau(t)=2 \partial \tau(t) / \partial t_{j p}=0, \quad j=1,3,5, \cdots
$$

which is just the condition $L^{p}=\left(L^{p}\right)_{+}$for $p$-reduced BKP hierarchy. Thus we have

$$
\mathbf{W}_{n}^{(1)} \tau(t)=W_{(2 n+1) p}^{(1)} \tau(t)=0, \quad n \geq 0
$$

For $m=1$, (26) shows that $Z_{2 k p}^{(2)} \tau=c_{k}^{(2)}(p) \tau$ with $k \geq-1$ where

$$
Z_{2 k p}^{(2)}=2 \sum_{n=0}^{k p-1} \partial_{2 n+1} \partial_{2 k p-2 n-1}+2 \sum_{n=0}^{\infty}(2 n+1) t_{2 n+1} \partial_{2 k p+2 n+1}
$$

for $k \geq 0$ and

$$
\begin{aligned}
Z_{2 k p}^{(2)}= & \frac{1}{2} \sum_{n=0}^{-k p-1}(2 n+1)(-2 k p-2 n-1) t_{2 n+1} t_{-2 k p-2 n-1} \\
& +2 \sum_{n=0}(2 n+1-2 k p) t_{2 n+1-2 k p} \partial_{2 n+1}
\end{aligned}
$$

for $k<0$. Define $l_{k}=Z_{2 k p}^{(2)} / 4 p$ with $k \geq-1$ then $l_{k}$ satisfy centerless Virasoro algebra $\left[l_{n}, l_{m}\right]=(n-m) l_{n+m}$ except that

$$
\left[l_{1}, l_{-1}\right]=2\left(l_{0}+\frac{1}{16 p}+\frac{p^{2}-1}{24 p}\right) .
$$


This means that the constants $c_{k \neq 0}^{(2)}(p)=0$ and $c_{0}^{(2)}(p)=-\left(1 / 4+\left(p^{2}-1\right) / 6\right)$. If we redefine a new set of operators as

$$
\mathbf{W}_{n}^{(2)} \equiv \mathbf{L}_{n}=l_{n}+\delta_{n, 0}\left(\frac{1}{16 p}+\frac{p^{2}-1}{24 p}\right), \quad n \geq-1
$$

then we have $\left[\mathbf{L}_{n}, \mathbf{L}_{m}\right]=(n-m) \mathbf{L}_{n+m}$ for $m, n \geq-1$. Therefore, the Virasoro constraint becomes

$$
\mathbf{W}_{n}^{(2)} \tau(t)=0, \quad n \geq-1
$$

In particular, the lowest Virasoro constraint $\mathbf{L}_{-1} \tau=0$ is given by

$$
\left(\frac{1}{2} \sum_{n=0}^{p-1}(2 n+1)(2 p-2 n-1) t_{2 n+1} t_{2 p-2 n-1}+2 \sum_{n=0}(2 n+1+2 p) t_{2 n+1+2 p} \partial_{2 n+1}\right) \tau=0
$$

which is just the pre-string equation (23). Moreover, when $n$ extends to all integers, $\mathbf{W}_{n}^{(2)}$ indeed constitute the generators of a standard Virasoro algebra with central charge $c=p$, namely,

$$
\left[\mathbf{L}_{n}, \mathbf{L}_{m}\right]=(n-m) \mathbf{L}_{n+m}+\delta_{n+m, 0} \frac{n^{3}-n}{12} p .
$$

For $m=2$, we may define the spin-3 generators as $\mathbf{W}_{n}^{(3)}=Z_{(2 n+1) p}^{(3)}$ which indeed satisfy the commutation relation

$$
\left[\mathbf{L}_{n}, \mathbf{W}_{m}^{(3)}\right]=(2 n-m) \mathbf{W}_{n+m}^{(3)}
$$

and the constraint equation

$$
\mathbf{W}_{n}^{(3)} \tau(t)=0, \quad n \geq-2 .
$$

The higher-spin generators can be treated in a similar manner.

\section{Grassmannian description of the string equation}

In this section we like to give a geometric description of the string equation for the $p$-reduced BKP hierarchy. Let $H$ be a Hilbert space defined by formal power series in $z$ that can be decomposed into two infinite-dimensional subspaces as $H=H_{+} \oplus H_{-}$where

$$
H_{+}=\operatorname{span}\left\{z^{0}, z^{1}, z^{2}, \cdots\right\}, \quad H_{-}=\operatorname{span}\left\{z^{-1}, z^{-2}, z^{-3}, \cdots\right\} .
$$

The Grassmannian $G r$ is defined by the set of all subspaces $V \subset H$ with the following conditions: $[19,22$ ]

$$
G r=\left\{V\left|V \subset H, p_{+}\right|_{V}: V \rightarrow H_{+} \text {(Fredholm), }\left.p_{-}\right|_{V}: V \rightarrow H_{-} \text {(compact) }\right\}
$$


where $p_{ \pm}$are projection operators. If $\left.p_{+}\right|_{V}: V \rightarrow H_{+}$is a bijection, then $V$ is called transversal to $H_{-}$, or transversal for short (i.e. $V$ belongs to the big cell $G r_{0} \subset G r$ ). The KP hierarchy can be regarded as a simple dynamical system on $G r$ (see [19] for the detail).

The BKP hierarchy is defined by the subvariety $G r^{B} \subset G r$ (see e.g. [23]) so that an infinitedimensional plane $V^{0} \in G r^{B}$ can be represented as follows [19, 22]:

$$
\begin{aligned}
V^{0} & =\operatorname{span}\left\{\left.\omega(t, z)\right|_{t=0},\left.\partial_{x} \omega(t, z)\right|_{t=0},\left.\partial_{x}^{2} \omega(t, z)\right|_{t=0}, \cdots\right\} \\
& =\operatorname{span}\left\{\omega(t, z), \text { for all } t \in \mathbb{C}^{\infty}\right\} .
\end{aligned}
$$

where $t=\left(t_{1}, t_{3}, t_{5}, \cdots\right)$ and $\omega(t, z)$ satisfies the bilinear equation (8). For the $p$-reduced BKP hierarchy, $\partial_{p} W=-\left(L^{p}\right)_{-} W$ and the wave function $\omega(t, z)$ associated with $V^{0}$ satisfies

$$
\partial_{p} \omega(t, z)=\left(L^{p}\right)_{+} \omega(t, z)=L^{p} \omega(t, z)=z^{p} \omega(t, z) \in V^{0}
$$

Therefore

$$
z^{p} V^{0} \subset V^{0} .
$$

On the other hand, the string equation (17) can be traced back to the Sato equation of the additional flow $\hat{\partial}_{1,1-2 p} W=-\left(A_{1,1-2 p}\right)_{-} W=0$ which follows

$$
\hat{\partial}_{1,1-2 p} \omega(t, z)=-\left(A_{1,1-2 p}\right)_{-} \omega(t, z)=0
$$

and hence

$$
\begin{aligned}
Q \omega(t, z) & =\frac{1}{2 p}\left(M L^{1-2 p}-p L^{-2 p}\right) \omega(t, z) \\
& =\frac{1}{2 p}\left(z^{1-2 p} \partial_{z}-p z^{-2 p}\right) \omega(t, z) \\
& \equiv A_{2 p} \omega(t, z) \in V^{0} .
\end{aligned}
$$

Therefore, the plane $V^{0} \in G r^{B}$ associated with $\omega(t, z)$ of $p$-reduced BKP hierarchy constrained by the string equation $\left[L^{p}, P\right]=1$ is invariant under the action of differential operators $L^{p}$ and $Q \equiv \frac{1}{2 p}\left(M L^{1-2 p}-p L^{-2 p}\right)$. They act as $z$-operators:

$$
L^{p} \mapsto z^{p}, Q \mapsto A_{2 p} \equiv z^{p} \frac{d}{d z^{2 p}} z^{-p}
$$

and

$$
z^{p} V^{0} \subset V^{0}, A_{2 p} V^{0} \subset V^{0} \text {, with }\left[A_{2 p}, z^{2 p}\right]=1 .
$$

The above discussions enable us to transform the original problem for solving the solution of the $p$-reduced BKP hierarchy constrained by the string equation to that described in $z$-operators in the context of Grassmannian. 


\section{Concluding Remarks}

We have investigated the string equation of the BKP hierarchy from additional symmetries point of view. We show that the $p$-reduced BKP hierarchy constrained by the string equation can be formulated in terms of Lax and Orlov-Schulman operators as what has been done for the KP hierarchy. In particular, the invariance of the additional symmetry with respect to the $\hat{t}_{1,1-2 p}$-flow is crucial for obtaining the string equation which together with the Adler-Shiotavan Moerbeke formula implies the $W$-constraints of the $\tau$ function. Furthermore, we show that the Lax-Orlov-Schulman formulation of the $p$-reduced BKP hierarchy with string equation can be transformed to that of Sato Grassmannian in terms of the spectral parameter $z$. In view of the works by Kac and Schwarz[14, 21], the Grassmannian description might provide a starting point for investigating the existence problem of BKP solutions characterized by the string equation. We hope to address this issue in our future work.

\section{Acknowledgments}

We like to thank N.C. Lee for helpful discussions. This work is supported by the National Science Council of Taiwan under Grant NSC97-2112-M-194-002-MY3.

\section{References}

[1] M. Adler and P. van Moerbeke, A matrix integral solution to two-dimensional Wp-gravity, Comm. Math. Phys. 147(1992)25-56

[2] M. Adler, T. Shiota and P. van Moerbeke, From the $w_{\infty}$-algebra to its central extension: a $\tau$-function approach. Phys. Lett. A 194 (1994), 33-43.

[3] M. Adler, T. Shiota and P. van Moerbeke, A Lax representation for the vertex operator and the central extension, Comm. Math. Phys. 171 (1995) 547.

[4] E. Date, M. Jimbo, M. Kashiwara and T. Miwa, Transformation groups for soliton equations, in Nonlinear integrable systems - classical theory and quantum theory, ed. by M. Jimbo and T. Miwa, (1983) 39-120, World Scientific.

[5] L.A. Dickey, Additional symmetry of KP, Grassmannian, and the string equation, Mod. Phys. Lett. A8,13(1993) 1259-1272.

[6] L.A. Dickey, On additional symmetries of the KP hierarchy and Sato's Bäcklund transformation, Comm. Math. Phys. 167 (1995), 227-233. 
[7] L.A. Dickey, Soliton equations and Hamiltonian systems. Second edition, World Scientific Publishing Co.

[8] R. Dijkgraaf, Intersection theory, integrable hierarchies and topological field theory, in New symmetry principles in quantum field theory (Cargese, 1991), 95-158, NATO Adv. Sci. Inst. Ser. B Phys., 295, Plenum, New York, 1992.

[9] R. Dijkgraaf, H. Verlinde, and E. Verlinde, Loop equations and Virasoro constraints in nonperturbative two-dimensional quantum gravity, Nuclear Phys. B 348 (1991), 435-456.

[10] M. Fukuma, H, Kawai and R. Nakayama, Infinite dimensional structure of two- dimensuinal quantum gravity, Comm. Math. Phys. 143 (1992) 371-403

[11] J. Goeree, $W$-constraints in 2D quantum gravity, Nuclear Phys. B 358 (1991), 737-757.

[12] J.S. He, K.L. Tian, A. Foerster, and W.X Ma, Additional symmetries and string equation of the CKP hierarchy, Lett. Math. Phys. 81 (2007) 119-134.

[13] C. Itzykson, J.B. Zuber, Combinatorics of the modular group. II: The Kontsevich integrals, Internat. J. Modern Phys. A 7 (1992) 5661-5705.

[14] V. Kac and A. Schwarz, Geometric interpretation of the partition function of 2D gravity. Phys. Lett. B 257 (1991) 329-334.

[15] M. Kontsevich, Intersection theory on the moduli space of curves and the matrix Airy function, Comm. Math. Phys. 147 (1992), 1-23.

[16] H.S. La, Symmetries in nonperturbative 2-d quantum gravity. Modern Phys. Lett. A 6 (1991), 573-580.

[17] H.S. La, Geometry of Virasoro constraints in nonperturbative 2-d quantum gravity. Comm. Math. Phys. 140 (1991), 569-588.

[18] A.Yu. Orlov and E.I. Schulman: Additional symmetries for integrable systems and conformal algebra repesentation, Lett. Math. Phys. 12 (1993), 171.

[19] M. Sato and Y. Sato, Soliton equations as dynamical systems on infinite-dimensional Grassmann manifold. Nonlinear partial differential equations in applied science (Tokyo, 1982), 259-271, North-Holland Math. Stud., 81, North-Holland, Amsterdam, 1983.

[20] K. Sawada and T. Kotera: A method for finding $N$-soliton solutions of the KdV equation and KdV-like equation. Progr. Theoret. Phys. 51 (1974), 1355-1367. 
[21] A. Schwarz, On solutions to the string equation. Modern Phys. Lett. A6 (1991), 2713-2725.

[22] G. Segal and G. Wilson, Loop groups and equations of KdV type, Inst. Hautes Etudes Sci. Publ. Math. 61 (1985), 5-65.

[23] T. Shiota, Prym varieties and soliton equations, in Infinite dimensional Lie algebras and groups, ed. V.G.Kac, Adv. Ser. in Math. Phys. 7, World Sci. (1989) 407-448.

[24] K. Takasaki: Quasi-classical limit of BKP hierarchy and W-infinity symmetries, Lett. Math. Phys. 28 (1993), 177-185.

[25] M.H. Tu, On the BKP hierarchy: Additional symmetries, Fay identity and Adler-Shiotavan Moerbeke formula, Lett. Math. Phys. 81(2007)91-105

[26] J. van de Leur, The Adler-Shiota-van Moerbeke formula for the BKP hierarchy, J. Math. Phys. 36 (1995) 4940.

[27] J. van de Leur, The $n$-th reduced BKP hierarchy, the string equation and $B W_{1+\infty^{-}}$constraints, Acta Appl.Math. 44(1996) 185-206.

[28] P. van Moerbeke, Integrable fundations of string theory, in Lectures on Integrable systems, Ed. O. Babelon, P. Cartier, Y. Kosmann-Schwarzbach, World Sci.(1994) pp.163-267

[29] E. Witten, Two-dimensional gravity and intersection theory on moduli space, Surveys in differential geometry (Cambridge, MA, 1990), 243-310

[30] E. Witten, On the Kontsevich model and other models of two-dimensional gravity, Proceedings of the XXth International Conference on Differential Geometric Methods in Theoretical Physics, 176-216, World Sci. Publ., River Edge, NJ, 1992. 